\title{
NOUVELLE
}

\section{SUMO module la stabilité et l'activation de PKR}

Faten El Asmi, Ghizlane Maarifi, Mohamed Ali Maroui, Laurent Dianoux, Mounira K. Chelbi-Alix

Inserm UMR-S 1124, université Paris Descartes, 45, rue des Saints-Pères, 75006 Paris, France. mounira.chelbi-alix@parisdescartes.fr

\section{SUMO}

SUMO (small ubiquitin modifier) est une protéine de 12 kDa qui appartient à la famille des UBL (ubiquitin-like proteins). Chez l'homme, il existe 5 paralogues ${ }^{1}$ de SUMO, les plus étudiés étant SUMO1, SUMO2 et SUMO3 qui sont ubiquitaires. SUMO2 et SUM03 possèdent $95 \%$ de séquences identiques, mais seulement $50 \%$ d'identité avec la séquence de SUMO1 [1]. Les protéines SUMO2 et SUM03 peuvent donc être reconnues par un même anticorps anti-SUM02/3. Les protéines SUMO2 et SUMO3 possèdent le motif consensus $\Psi K x \varepsilon^{2}$ qui leur permet de s'oligomériser et de former des chaînes poly-SUM02/3. Ce motif n'est pas présent dans la protéine SUMOl qui n'est donc impliquée que dans la terminaison des chaînes poly-SUMO. Les polychaînes formées par SUM02/3 reconnaissent une SUMO ubiquitine ligase nommée RNF4 (ring finger protein 4), qui provoque l'ubiquitination et, par suite, la dégradation de substrats conjugués à SUM02/3 en réponse à différents stimulus tels que le trioxyde d'arsenic ou les interférons $\alpha$ et $\gamma[2](\rightarrow)$.

$\rightarrow$ Voir la Nouvelle de G. Maarifi et al., $m / s n^{\circ} 2$, février 2016, page 141

D'autres différences portent sur le niveau d'expression de ces paralogues

${ }^{1}$ Produits de gènes homologues issus d'un même gène ancestral

$2 \Psi$ représente un acide aminé hydrophobe, $K$ la lysine qui forme la liaison isopeptidique avec le SUMO, $x$ un acide aminé quelconque, et $\varepsilon$ un acide aminé acide (aspartique ou glutamique). ainsi que sur leur susceptibilité aux SUMO protéases ou déSUMOylases.

La SUMOylation est une modification réversible qui implique la liaison covalente isopeptidique entre un résidu glycine dans la région C-terminale de SUMO et une lysine de la protéine substrat. Ce processus est très proche biochimiquement de celui de l'ubiquitination, notamment en impliquant une cascade d'enzymes similaires, mais également d'autres qui lui sont propres [3] $(\rightarrow)$.

$(\rightarrow)$ Voir la Synthèse de $F$. Lehembre et $A$. Dejean, $m / s n^{\circ} 11$, novembre 2000, page 1246

Ce processus met en jeu une série de trois étapes enzymatiques: la première étape correspond au clivage de la partie C-terminale de la protéine SUMO ; assurée par l'enzyme d'activation $\varepsilon l$, elle engendre l'activation de SUMO et l'exposition du dipeptide de glycines (GG). La deuxième étape consiste en la création d'un lien entre SUMO-GG et la cystéine catalytique de l'enzyme $\varepsilon l$. La troisième étape est la conjugaison du SUMO-GG, qui implique I'unique enzyme de conjugaison UBC9 (ubiquitin-like conjugating enzyme 9). La SUMOylation est un processus dynamique entre les activités opposées des enzymes de conjugaison ( $\varepsilon 1, \varepsilon 2, \varepsilon 3)$ et celles des enzymes de déconjugaison (déSUMOylases, SENP [sentrin-specific-protease]). Elle est impliquée dans de nombreux processus cellulaires tels que la régulation transcriptionnelle, la dégradation des protéines et la défense antivirale [4].

L'expression exogène stable de SUMOl ou de SUM03 dans des cellules humaines réduit la phosphorylation de STATl (signal transducer and activator of transcription 1) induite par l'interféron gamma (IFN- $\gamma$ ), inhibant ainsi la transcription. La phosphorylation de STATl en réponse à I'IFN- $\alpha$, est également réduite, mais la transcription n'est

$(\rightarrow)$ Voir la Nouvelle de G. Maarifi et al., $m / s n^{\circ} 2$, février 2016, page 141 alors pas altérée [2] $(\rightarrow)$.

Récemment, notre équipe a montré que le facteur de restriction MxA ${ }^{3}$ (Myxovirus resistance $A$ ) est conjugué à SUMO par la lysine 48 [5], qu'il est fortement stabilisé dans les cellules qui expriment SUMO1 ou SUMO3 et qu'il relaye l'effet antiviral (anti-VSV [vesicular stomatitis virus]) de SUMO [6]. Cependant, la spécificité des différents paralogues de SUMO restait à élucider.

\section{PKR et les différents paralogues} de SUMO

Comme MxA, la protéine PKR (doublestranded RNA-activated protein kinase) est un facteur de restriction induit en réponse à I'IFN- $\alpha$. Identifiée en 1976, PKR est une sérine/thréonine protéinekinase de $68 \mathrm{kDa}$ qui joue un rôle clé dans divers processus cellulaires, en particulier dans la défense antivirale [7]. Cette protéine possède deux domaines de liaison à l'ARN double brin ( $d b)$, un domaine d'autophosphorylation et de dimérisation, ainsi qu'un domaine kinase de reconnaissance des sérines et thréonines.

\footnotetext{
${ }^{3} M x A$ est induit par les interférons de type I (IFN- $\alpha$ et IFN- $\beta$ ).
} 


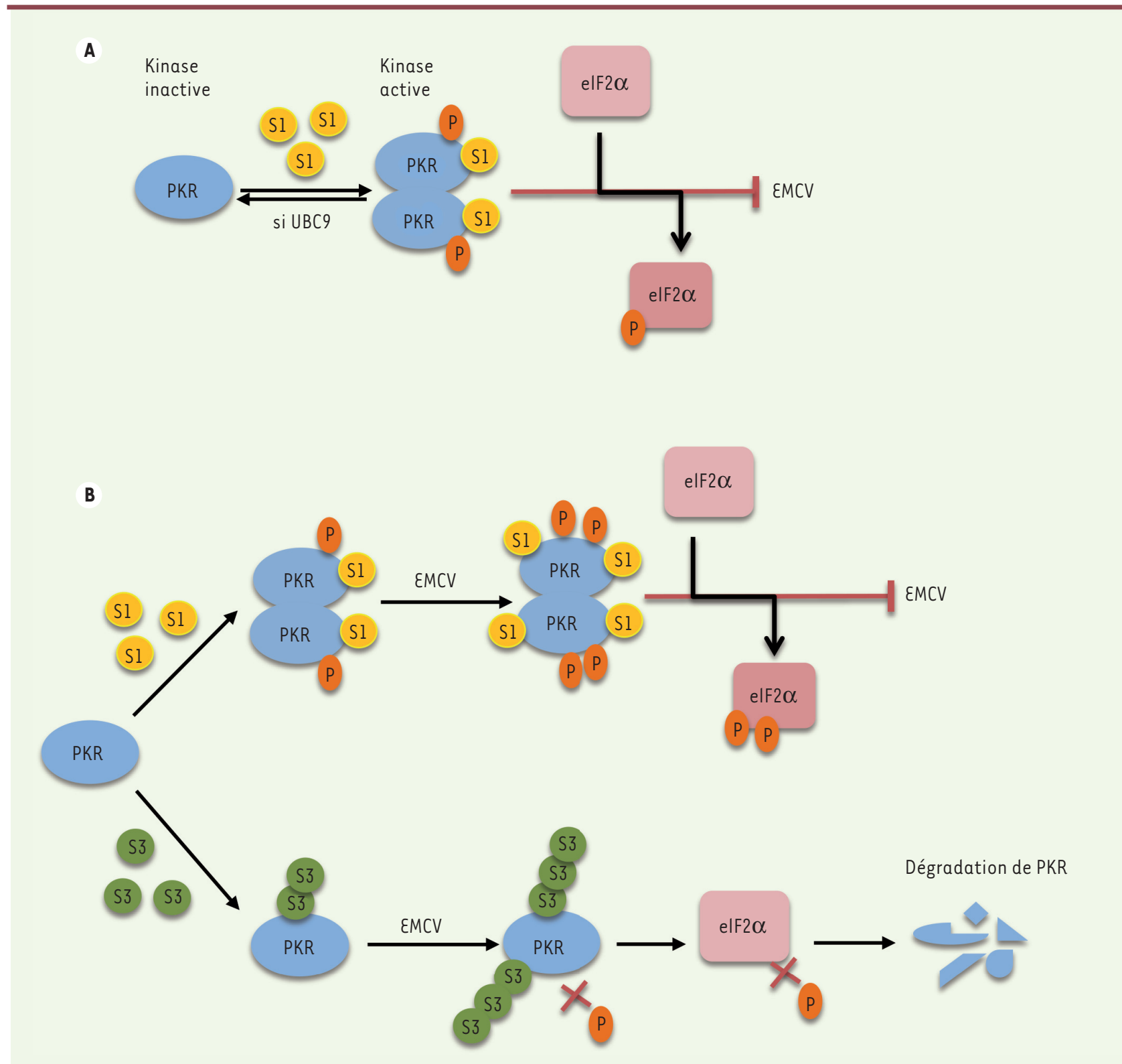

Figure 1. Effet de SUMO1 et SUMO3 sur la voie PKR/elF2 $\alpha$. A. L'expression de SUMO1 induit l'activation de PKR et de l'elF2 $\alpha$, ce qui conduit à l'inhibition de l'EMCV. Ce processus est inversé quand les cellules qui expriment SUMOl sont dépourvues d'UBC9 (Ubiquitin-like conjugating enzyme 9) par siARN (small interfering ARN). B. L'infection par l'EMCV induit la conjugaison de PKR à SUM01, ce qui entraîne une plus forte activation de PKR et d'elF2 $\alpha$. À l'inverse, la conjugaison de PKR à SUM03 va bloquer sa phosphorylation et celle de l'elF-2 $\alpha$ et entraîner la dégradation de PKR par la voie des caspases.

La protéine PKR est ubiquitaire, elle est exprimée de façon constitutive en tant que monomère inactif et est induite, sous sa forme inactive, en réponse à I'IFN- $\alpha$. Elle est activée par autophosphorylation après sa liaison à des ARN$\mathrm{db}$ (des intermédiaires réplicatifs de nombreux virus), ce qui entraîne la for- mation de dimères actifs de l'enzyme (Figure 1A). Activée, PKR phosphoryle plusieurs substrats cellulaires, le plus étudié étant la sous-unité $\alpha$ du facteur d'initiation de la traduction (elF2 $\alpha$ ). La phosphorylation de l'elF2 $\alpha$ inhibe l'initiation de la traduction et donc, par exemple, la prolifération du virus dans une cellule infectée. L'importance de PKR dans la défense antivirale a été révélée par la mise en évidence que de nombreux virus ciblent cette kinase en la délocalisant, en la dégradant ou en produisant des inhibiteurs de cet enzyme. PKR est non seulement phosphorylée, mais elle est également la cible de 
I'ISGylation ${ }^{4}$ [8] et de la SUMOylation [9]. La kinase PKR mutée sur les trois sites de SUMOylation, correspondant aux lysines Lys-60, Lys-150 et Lys-440, a des activités réduites de liaison à l'ARN-db, de dimérisation et de phosphorylation de l'elF-2 $\alpha[9]$.

\section{Effet de SUMO1 et SUMO3 sur la localisation de PKR}

La protéine PKR est localisée dans le cytoplasme et le noyau. Ses activités sont principalement retrouvées au niveau du cytoplasme. Son rôle dans le noyau n'est pas encore élucidé. Par fractionnement cellulaire, nous avons montré que l'expression stable de SUMO1 ou de SUMO3 ne modifie pas la distribution de PKR entre le cytoplasme et le noyau. Néanmoins, les formes de PKR modifiées par SUMOI et SUM03 sont localisées majoritairement dans le noyau dans des cellules contrôles ou lors d'expressions exogènes de SUMO1 ou SUM03 [10]. Cependant, l'étude par immunofluorescence a révélé que dans les cellules qui expriment SUM03, la localisation de PKR est altérée dans le cytoplasme et le noyau en comparaison avec les cellules contrôles. Dans le cytoplasme, PKR est plus concentrée autour de la membrane périnucléaire. Dans le noyau des cellules contrôles, PKR a un aspect microponctué et l'expression de SUM03 la localise dans de gros corps (nuclear dots). [10].

\section{Effets différentiels de SUMO1 et SUM03 sur l'activation de PKR}

Fait remarquable, l'expression stable de SUMOl dans les cellules de la lignée humaine HeLa active la protéine PKR et le facteur elF-2 $\alpha$. Les phosphorylations induites sont inhibées quand l'expression de l'unique enzyme de conjugaison UBC9 est diminuée (Figure IA). Inversement, elles sont augmentées quand les cellules sont transfectées par de I'ARN$\mathrm{db}$, l'acide polyinosinique-polycytidy-

\footnotetext{
${ }^{4}$ Le produit de I'ISGI5 (interferon-stimulated gene 15) est une protéine de $17 \mathrm{kDa}$ qui se lie de façon covalente aux protéines cytoplasmiques et nucléaires. Ce processus nommé ISGylation est semblable à l'ubiquitination.
}

lique ${ }^{5}-$ poly $(I: C)$-, ou infectées par les virus de la stomatite vésiculaire (VSV) ou de l'encéphalomyocardite (EMCV) (Figure 1B). L'activation de PKR et elF2 $\alpha$ par SUMOl est corrélée à une inhibition de la multiplication de l'EMCV [10], mais n'a pas effet sur le VSV, l'action de SUMO sur ce virus étant relayée par la protéine MxA et non PKR [6]. L'expression de SUMO3 a un effet opposé à celui de SUMO1. Elle n'influe pas directement sur PKR et l'elF-2 $\alpha$, mais elle bloque leur phosphorylation induite après transfection par le poly (I:C) ou à la suite de l'infection par le VSV ou l'EMCV [10] (Figure 1B).

\section{Effets différentiels de SUMO1}

\section{et SUM03 sur la stabilité de PKR}

L'EMCV augmente, dès 2 heures après l'infection, la SUMOylation de plusieurs protéines dans la cellule, et particulièrement la conjugaison de PKR à SUMOl et à SUM03 [10]. II faut noter que l'infection par l'EMCV n'altère pas la SUMOylation d'autres protéines telles que STATl, connue pour avoir un site de conjugaison à SUMO. Ce processus va entraîner une plus forte activation de la voie PKR/ elF-2 $\alpha$ dans le cas de SUM01. Par contre, l'augmentation induite par l'EMCV de la modification de PKR par SUM03, provoque la dégradation de PKR via la voie des caspases [10]. Un travail précédent avait montré que l'infection par l'EMCV augmentait la SUMOylation de PMLIII (promyelocytic leukemia protein III, un autre facteur de restriction) entraînant sa dégradation via le protéasome [11]. Ces résultats révèlent les stratégies développées par l'EMCV pour inhiber l'action antivirale de PML et de PKR.

\section{Conclusions}

L'activation de PKR et d'elF2 $\alpha$ par SUMO1 en l'absence d'infection virale est un gain de fonction de PKR et suggère un nouveau mécanisme d'activation de la

\footnotetext{
${ }^{5}$ L'acide polyinosinique-polycytidylique est un ARN double brin synthétique, structurellement semblable à l'ARN bicaténaire de certains virus.
}

voie PKR/elF2 $\alpha$. Cette activation est également amplifiée par une infection virale. II est intéressant de noter que I'ISGylation de PKR sur les lysines Lys-69 et Lys-159 par I'ISG15, une autre UBL (ubiquitin-like protein), aboutit également à l'activation de PKR [8].

À l'inverse de SUM01, SUM03 contrecarre l'activation de PKR et celle de l'elF2 $\alpha$ induite par les virus VSV et EMCV (Figure 1B). L'EMCV a un double effet sur PKR en induisant sa conjugaison par SUMOl et SUM03. La modification par SUMOl va induire son activation et celle du facteur elF-2 $\alpha$, entraînant l'inhibition du virus. Inversement, la SUMOylation de PKR par SUM03 provoquée par l'EMCV induit la dégradation de PKR et s'oppose ainsi à son pouvoir antiviral. Ce virus a donc développé une stratégie pour détourner la machinerie cellulaire à son profit en dégradant la protéine PKR.

L'ensemble de ces résultats montre que les paralogues de SUMO, SUMOl et SUM03, ont des effets différents sur la localisation, la stabilité et l'activation de la protéine PKR. Ces données font entrevoir des perspectives nouvelles pour l'étude de la modification par SUMO de PKR ainsi que d'autres facteurs de restriction. $\diamond$

Modulation by SUMO of PKR activation and stability

\section{LIENS D'INTÉRÊT}

Les auteurs déclarent n'avoir aucun lien d'intérêt concernant les données publiées dans cet article.

\section{RÉFÉRENCES}

1. Johnson ES. Protein modification by SUMO. Annu Rev Biochem $2004 ; 73: 355-82$

2. Maarifi G, Dianoux L, Nisole S, Chelbi-Alix MK. Les paralogues de SUMO et la réponse interféron. Med Sci (Paris) $2016 ; 32: 141-3$.

3. Lehembre F, Dejean A. SUMO : une nouvelle voie de modification des protéines apparentée à l'ubiquitinylation. Med Sci (Paris) $2000 ; 16$ : 1242-6.

4. Hannoun Z, Maarifi G, Chelbi-Alix MK. The implication of SUMO in intrinsic and innate immunity. Cytokine Growth Factor Rev 2016 ; 29 : 3-16.

5. Brantis-de-Carvalho CE, Maarifi G, Goncalves Boldrin $P E$, et al. MxA interacts with and is modified by the SUMOylation machinery. Exp Cell Res 2015 ; 330 : 151-63.

6. Maarifi G, Hannoun Z, Geoffroy MC, et al. MxA mediates SUMO-induced resistance to vesicular stomatitis virus. J Virol 2016 ; 90 : 6598-610. 


\section{RÉFÉRENCES}

7. Sadler AJ, Williams BR. Structure and function of the protein kinase R. Cur Top Microbiol Immunol 2007 ; $316: 253-92$.

8. Okumura F, Okumura AJ, Uematsu K, et al. Activation of double-stranded RNA-activated protein kinase (PKR) by interferon-stimulated gene 15 (ISG15) modification down-regulates protein translation. J Biol Chem 2013 ; $288: 2839-47$

9. de la Cruz-Herrera CF, Campagna M, Garcia MA, et al. Activation of the double-stranded RNA-dependent protein kinase PKR by small ubiquitin-like modifier (SUMO).J Biol Chem 2014 ; 289 : 26357-67.
10. Maarifi G, દl Asmi F, Maroui MA, et al. Differentia effects of SUMOI and SUMO3 on PKR activation and stability. Sci Rep 2018 ; 8 : 1277

11. El McHichi B, Regad T, Maroui MA, et al. SUMOylation promotes PML degradation during encephalomyocarditis virus infection. J Virol $2010 ; 84$ : 11634-45.

\section{NOUVELLE}

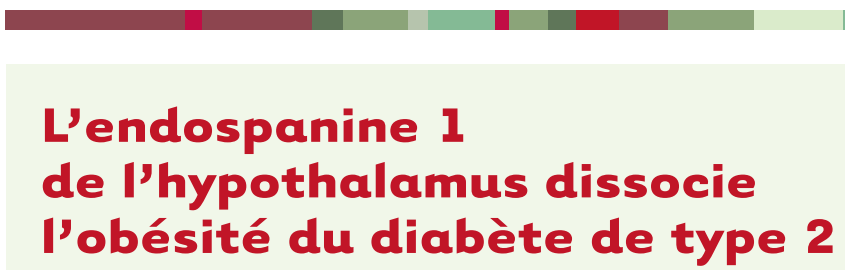

Clara Roujeau, Ralf Jockers, Julie Dam

> L'obésité est un problème majeur de santé publique. Elle touchait en effet $12 \%$ de la population mondiale en 2017 [1]. Cette maladie chronique, caractérisée par un déséquilibre de la balance énergétique conduisant à un excès de masse grasse, est un facteur de risque pour de nombreuses pathologies telles que le diabète de type 2 (DT2), les maladies cardiovasculaires et les cancers. De nouvelles cibles thérapeutiques, qui tendent à restaurer le maintien de la balance énergétique, sont toujours activement recherchées afin d'enrayer cette épidémie qu'est devenue l'obésité. En 1994, la découverte de la leptine, hormone anorexigène ${ }^{l}$ primordiale pour le contrôle des homéostasies énergétique et glucidique, a fait émerger de grands espoirs [2] $(\rightarrow)$.

$\rightarrow$ Voir la Nouvelle de C. Vatier et al., $\mathrm{m} / \mathrm{s}$ $\varepsilon$ n effet, l'adminis$n^{\circ} 10$, octobre 2010 , page 803

tration de leptine à des patients obèses et diabétiques présentant une déficience congénitale en leptine permet de diminuer de façon drastique leur poids corporel par normalisation de la prise alimentaire et de corriger leur DT2 [3].

${ }^{1}$ hormone qui diminue l'appétit par opposition aux signaux oréxigènes qui le stimulent.
Toutefois, cette déficience congénitale en leptine est rare. La majorité des patients obèses est, au contraire, incapable de répondre aux signaux anorexigènes de la leptine, malgré des taux très élevés de leptine circulante, indiquant ainsi chez ces patients, un état de «résistance à la leptine» [4]. Prévenir et/ou lever la résistance à la leptine constituent donc un défi majeur pour la recherche dans le domaine de l'obésité [4].

\section{Leptine, OBR et Endol :}

un trio impliqué dans le contrôle

des homéostasies énergétique

et glucidique

La leptine est une hormone synthétisée et sécrétée par le tissu adipeux. Son action anorexigène est relayée, notamment, par la stimulation de neurones du noyau arqué de l'hypothalamus (ARC) après activation de son récepteur membranaire OBR (obesity receptor) $[5](\rightarrow)$.

$(\rightarrow)$ Voir la Nouvelle de $\varepsilon$. Balland et V. Prévot, $\mathrm{m} / \mathrm{s} \mathrm{n}^{\circ}$ 6-7, juinL'expression de surface d'OBR est cruciale pour la sensibilité neuronale à la leptine. L'OBR stimule l'activation de voies de signalisation intracellulaire spécifiques dans les neurones de I'ARC. Parmi celles-ci, la voie JAK2
Inserm U1016, CNRS UMR 8104, université Paris Descartes, Sorbonne Paris Cité, Équipe pharmacologie fonctionnelle et physiopathologie des récepteurs membranaires, Institut Cochin, 22, rue Méchain, 75014 Paris, France. julie.dam@inserm.fr

(Janus kinase 2)/STAT3 (signal transducer and activator of transcription 3) apparaît nécessaire pour le contrôle de I'homéostasie énergétique par la leptine, qui active l'expression de signaux anorexigènes et inhibe celle de signaux orexigènes [6]. La voie PI3K (phosphatidylinositide 3-kinase)/AKT (proteine kinase B) est, quant à elle, impliquée dans le maintien de l'homéostasie glucidique [7].

Plusieurs mécanismes sont à l'origine de l'établissement de la résistance à la leptine, dont un défaut de localisation du récepteur OBR à la surface des neurones, limitant ainsi leur sensibilité à I'hormone [4]. Le nombre de récepteurs exprimés à la membrane des cellules détermine l'intensité de la signalisation induite par la leptine. En recherchant les acteurs moléculaires contribuant possiblement à la résistance des cellules à la leptine, nous avons identifié une protéine, l'Endospanine l (Endol), comme étant un régulateur négatif de l'exposition du récepteur OBR à la surface des cellules. Endol interagit avec OBR. Elle le séquestre dans les compartiments intracellulaires et favorise sa dégradation par le lysosome. Endol diminue donc la quantité de récepteurs 